\title{
Е.С. Сухачева
}

\section{О ФУНКЦИЯХ ПЕРВОГО КЛАССА БЭРА НА НЕКОТОРЫХ КЛАССАХ НЕМЕТРИЗУЕМЫХ ПРОСТРАНСТВ ${ }^{1}$}

\begin{abstract}
Для функций первого класса Бэра заданных на метризуемых пространствах известен критерий Бэра. Доказывается аналог этой теоремы для функций первого класса Бэра, заданных на более широком классе пространств, а именно на пространствах, являющихся одновременно наследственно линделефовыми и наследственно бэровским, но не обязательно метризуемых. В частности, прямая Зоргенфрея, ее модификции и пространства Хаттори обладают этими свойствами.
\end{abstract}

Ключевые слова: прямая Зоргенфрея, функиия первого класса Бэра, наследственно бэровское пространство, наследственно линделефово пространство, cl-функиия, множества типа $F_{\sigma} и G_{\delta}$.

В данной работе все топологические пространства подразумеваются нормальными и используются следующие обозначения: $\mathbb{N}$ - множество натуральных чисел; $\mathbb{R}$ - пространство вещественных чисел, наделенное стандартной евклидовой топологией; символом $S$ обозначается прямая Зоргенфрея (или «стрелка»), представляющая собой множество вещественных чисел, топология в котором порождена базой $\{(a, b]: a, b \in \mathbb{R}, a<b\}$. Если множество $A \subset \mathbb{R}$, то символом $S_{A}$ обозначим топологическое пространство, в котором база окрестностей точки $x$ определяется следующим образом:

$$
\begin{aligned}
& \{[x, x+\varepsilon), \forall \varepsilon>0\}, \text { если } x \in A \subset \mathbb{R} ; \\
& \{(x-\varepsilon, x], \forall \varepsilon>0\}, \text { если } x \in \mathbb{R} \backslash A .
\end{aligned}
$$

В частности, если $A=\varnothing$, то $S_{A}=S$.

Множеством первой категории в топологическом пространстве называется множество, представимое в виде счетного объединения нигде не плотных множеств. Топологическое пространство $X$ называется бэровским, если пересечение любой последовательности открытых всюду плотных множеств всюду плотно. Топологическое пространство $X$ называется наследственно бэровским, если каждое замкнутое подмножество $F \subset X$ является бэровским пространством.

В работе [3] показано, что регулярное, с первой аксиомой счетности пространство является наследственно бэровским тогда и только тогда, когда оно не имеет счетного замкнутого подпространства без изолированных точек.

Предложение 1. Пространство $S_{A}$ является наследственно бэровским для любого множества $A \subset \mathbb{R}$.

Доказательство. Поскольку пространство $S_{A}$ регулярное и имеет первую аксиому счетности, то в силу предыдущего утверждения достаточно показать, что

\footnotetext{
${ }^{1}$ Исследование выполнено при финансовой поддержке РФФИ в рамках научного проекта № 17-5118051.
} 
пространство $S_{A}$ не имеет счетного замкнутого подпространства без изолированных точек. Пусть множество $F$ замкнуто в $S_{A}$ и не имеет изолированных точек. Тогда замыкание $\bar{F}$ множества $F$ в евклидовой топологии прямой также не содержит изолированных точек. Это означает [1], что множество $\bar{F}$ несчетно. Поскольку множество $\bar{F} \backslash F$ счетно, то $F$ является несчетным.

Для любого множества $A \subset \mathbb{R}$, пространство $S_{A}$ нормальное и линделефовое. Эти факты можно доказать аналогично доказательствам нормальности и линделефовости прямой Зоргенфрея [8].

Совершенным пространством называется пространство, в котором все замкнутые множества имеют тип $G_{\delta}$. Нетрудно видеть, что в пространстве $S_{A}$ открытые множества являются счетным объединением промежутков вида $\left(a_{1}, b_{1}\right],\left[a_{2}, b_{2}\right)$, $\left(a_{3}, b_{3}\right),\left[a_{4}, b_{4}\right]$, а каждый из этих промежутков имеет тип $F_{\sigma}$. Таким образом, пространство $S_{A}$ является совершенным для любого множества $A \subset \mathbb{R}$. Отсюда следует, что пространство $S_{A}$ наследственно линделефово [8].

Пусть $X$ - топологическое пространство. Отображение $f: X \rightarrow \mathbb{R}$ называется функиией первого класса Бэра, если существует последовательность непрерывных функций $\left\{f_{i}\right\}_{i=1}^{\infty}$, поточечно сходящаяся к функции $f$ на множестве $X$. Множество всех функций первого класса Бэра обозначается $B_{1}(X)$. В силу того, что топология, заданная на $S_{A}$, тоньше, чем топология на прямой, то $B_{1}(\mathbb{R}) \subset B_{1}\left(S_{A}\right)$, но обратное включение не верно.

Пример 2. Пусть $K$ - канторово множество в евклидовой прямой. Тогда $\mathbb{R} \backslash K=\bigcup_{n=1}^{\infty}\left(a_{n}, b_{n}\right)$. Пусть $B=\left\{b_{n}\right\}_{n \in \mathbb{N}}$ и для каждого натурального $n$ заданы возрастающие последовательности $\left\{b_{n}^{i}\right\}_{i \in \mathbb{N}}, b_{n}^{i} \in\left(a_{n}, b_{n}\right)$, сходящиеся к точкам $b_{n}$. Определим непрерывные функции $f_{i}: S \rightarrow \mathbb{R}$ следующим образом: $f_{i}(x)=0$, если $x \in(K \backslash B) \cup\left(\bigcup_{n=1}^{i}\left[a_{n}, b_{n}^{i}\right)\right), f_{i}\left(b_{n}\right)=1$ при $n \leq i$ и линейные на каждом множестве $\left[b_{n}^{i}, b_{n}\right]$. Нетрудно видеть, что для всех $i \in \mathbb{N}$ функции $f_{i}$ непрерывны на $S$ и $\lim _{i \rightarrow \infty} f_{i}(x)=\chi_{B}(x)$ для любой точки $x \in S$. Таким образом, характеристическая функция $\chi_{B}$ является функцией первого класса Бэра на $S$. Так как функция $\left.\chi_{B}\right|_{K}$ не имеет точек непрерывности на замкнутом в евклидовой топологии множестве $K$, то $\chi_{B} \notin B_{1}(\mathbb{R})$ по теорема Бэра [4].

Тот факт, что пространства $B_{1}(\mathbb{R})$ и $B_{1}(S)$ различны также следует из [6] ввиду того, что $B_{1}(\mathbb{R})$ секвенциально сепарабельно, а $B_{1}(S)$ - нет (напомним, что пространство $X$ называется секвенциально сепарабельным, если существует счетное подмножество $D \subset X$, такое, что любая точка $x \in X$ есть предел некоторой последовательности точек из множества $D$ ). 
Функцию $f$, заданную на топологическом пространстве $X$ со значениями в вещественной прямой, называют $c l$-функцией (cliquish) в точке $x \in X$, если для любого $\varepsilon>0$ и любой окрестности $U$ точки $x$ существует открытое непустое множество $G \subset U$, такое, что $|f(y)-f(z)|<\varepsilon$ для любых двух точек $y, z \in G$. Функцию $f$ называют $c l$-функцией, если она сl-функция в каждой точке $x \in X$, a множество всех таких функций обозначается $C L(X)$. В работе [5] показано, что для любой cl-функции $f$, заданной на топологическом пространстве $X$, множество точек разрыва $D_{f}$ функции $f$ является множеством первой категории. Из этого вытекает следующее

Предложение 3. Пусть $X$ - бэровское пространство, $f \in C L(X)$ и $C_{f}-$ множество точек непрерывности функции $f$. Тогда множество $C_{f}$ всюду плотно в $X$.

Доказательство. Поскольку $f \in C L(X)$, то в силу предыдущего утверждения $D_{f}=\bigcup_{n=1}^{\infty} F_{n}$, где множества $F_{n}$ нигде не плотны. Следовательно, $C_{f}=X \backslash D_{f}=$ $=X \backslash\left(\bigcup_{n=1}^{\infty} F_{n}\right)=\bigcap_{n=1}^{\infty}\left(X \backslash F_{n}\right)$. Тогда множества $X \backslash F_{n}$ открыты, всюду плотны и, поскольку пространство $X-$ бэровское, то множество $C_{f}$ всюду плотно.

Теорема 4. Пусть $X$ - бэровское пространство. Тогда $B_{1}(X) \subset C L(X)$.

Доказательство. Пусть множество $U$ открыто в $X$. Тогда подпространство $U$ - бэровское (см. [7]). Пусть функция $g \in B_{1}(X)$ и $f=\left.g\right|_{U}$. Очевидно, что $f \in B_{1}(U)$. Пусть последовательность непрерывных функций $\left\{f_{n}\right\}_{n \in \mathbb{N}}$ на множестве $U$ поточечно сходится к функции $f$. Следовательно, для любой точки $x \in U$ последовательность $\left\{f_{n}(x)\right\}_{n=1}^{\infty}$ является фундаментальной в $\mathbb{R}$. Зафиксируем $\varepsilon>0$. Тогда $U=\bigcup_{p \in N} F_{p}$, где $F_{p}=\bigcap_{n, m \geq p}\left\{x \in X ;\left|f_{n}(x)-f_{m}(x)\right| \leq \frac{\varepsilon}{3}\right\}$. Заметим, что $F_{p} \cap U-$ замкнуто в $U$ и $U=\bigcup_{p \in N}\left(F_{p} \cap U\right)$. Поскольку подпространство $U$ бэровское, то найдется номер $p_{0} \in \mathbb{N}$, такой, что $\operatorname{int}_{U}\left(F_{p_{0}} \cap U\right) \neq \varnothing$. Тогда множество $V=\operatorname{int}_{U}\left(F_{p_{0}} \cap U\right)$ открыто в $U$, следовательно, открыто в $X$ и $V \subset F_{p_{0}} \cap U$. Это означает, что для любых $n, m \geq p_{0}$ и точки $x \in V$ верно неравенство $\left|f_{n}(x)-f_{m}(x)\right| \leq \frac{\varepsilon}{3}$. Переходя к пределу при $m \rightarrow \infty$ получаем, что $\left|f_{n}(x)-f(x)\right| \leq \frac{\varepsilon}{3}$ для любой точки $x \in V$ и $n \geq p_{0}$. В силу непрерывности функции $f_{p_{0}}$ существует окрестность $W \subset V$, такая, что для любых $y, z \in W$ и $n \geq p_{0}$ верно неравенство $\left|f_{p_{0}}(y)-f_{p_{0}}(z)\right|<\varepsilon / 3$. Применяя неравенства треугольника, получаем $|f(y)-f(z)|<\varepsilon$. 
Из предложения 3 и теоремы 4 получаем

Следствие 5. Пусть $X$ - наследственно бэровское пространство и $f \in B_{1}(X)$. Тогда для любого замкнутого множества $F \subset X$ функция $\left.f\right|_{F}$ на множестве $F$ имеет точку непрерывности.

Доказательство следующих трех лемм повторяет доказательства этих утверждений для метризуемых пространств (см. [4]), если учесть тот факт, что пространство $X$ - совершенно нормальное. Мы приведем доказательство только последней из них.

Лемма 6. Пусть $X$ - топологическое пространство. Если последовательность функций первого класса Бэра $f_{n}: X \rightarrow \mathbb{R}$ сходится равномерно к функции $f: X \rightarrow \mathbb{R}$, то $f \in B_{1}(X)$.

Лемма 7. Пусть $X$ - совершенное пространство. Если $X=\bigcup_{i=1}^{n} A_{i}$, где множества $A_{i}$ имеют тип $F_{\sigma}$, то существуют попарно непересекающиеся множества $B_{1}, \ldots, B_{n}$ типа $F_{\sigma}$, такие, что $X=\coprod_{i=1}^{n} B_{i}$ и $B_{i} \subset A_{i}$ для любого $i=\overline{1, n}$.

Лемма 8. Пусть $X$ - нормальное пространство и $f: X \rightarrow\left\{c_{0}, \ldots, c_{n}\right\}$, где числа $c_{0}<\ldots<c_{n}$ из $\mathbb{R}$. Если множества $E_{k}=\left\{x \in X ; f(x)=c_{k}\right\}$ имеют тип $F_{\sigma}$ при $k=\overline{0, n}$, то $f \in B_{1}(X)$.

Доказательство. Пусть $E_{k}=\bigcup_{i=1}^{\infty} F_{i}^{k}$. Положим $L_{m}^{k}=\bigcup_{i=1}^{m} F_{i}^{k}, L_{m}=\bigcup_{k=1}^{n} L_{m}^{k}$ и определим функцию $\varphi_{m}: L_{m} \rightarrow \mathbb{R}$ следующим образом: $\varphi_{m}(x)=c_{k}$, если $x \in L_{m}^{k}$. Заметим, что все множества $L_{m}^{k}$ - непересекающиеся и замкнуты в $X$ и, следовательно, функция $\varphi_{m}$ непрерывная на $L_{m}$. Тогда в силу теоремы Титце - Урысона существует непрерывное продолжение $\widetilde{\varphi_{m}}: X \rightarrow \mathbb{R}$, такое, что $\widetilde{\varphi_{m}}(x)=\varphi_{m}(x)$ для любого $x \in L_{m}$. Нетрудно видеть, что $\lim _{m \rightarrow \infty} \widetilde{\varphi_{m}}(x)=f(x)$ для любого $x \in X$.

Для функций первого класса Бэра заданных на метризуемых пространствах, хорошо известны теорема Лебега и теорема Бэра. Следующие теоремы являются их аналогами в случае наследственно линделефовых пространств.

Теорема 9. Пусть $X$ - наследственно линделефово пространство и функция $f: X \rightarrow \mathbb{R}$. Функция $f \in B_{1}(X)$ тогда и только тогда, когда для любого открытого множества $U \subset \mathbb{R}$ его прообраз $f^{-1}(U)$ имеет тип $F_{\sigma}$.

Доказательство. Пусть множество $U$ открыто в $\mathbb{R}$. Тогда $U=\bigcup_{k=1}^{\infty} F_{k}$ для некоторых множеств $F_{k}$, замкнутых в $\mathbb{R}$ и таких, что $F_{k} \subset \operatorname{int}_{\mathbb{R}} F_{k+1}$ для $k \in N$. Поскольку $f \in B_{1}(X)$, то существует последовательность непрерывных функций 
$\left\{f_{n}\right\}_{n \in N}$, такая, что $f(x)=\lim _{n \rightarrow \infty} f_{n}(x)$. Тогда $f^{-1}(U)=\bigcup_{k=1}^{\infty} \bigcap_{n=k}^{\infty} f_{n}^{-1}\left(F_{k}\right)$ и, следовательно, множество $f^{-1}(U)$ имеет тип $F_{\sigma}$.

Предположим теперь, что для любого открытого множества $U \subset \mathbb{R}$ его прообраз $f^{-1}(U)$ имеет тип $F_{\sigma}$ и функция $f$ ограничена сверху и снизу точками $a$ и $b$ соответственно. Разобьём множество значений на конечное число промежутков точками $a=c_{0}<c_{1}<\ldots<c_{n}=b$ длины не больше $1 / n$ и положим $A_{0}=\left\{x \in X ; f(x)<c_{1}\right\}, \quad A_{k}=\left\{x \in X ; c_{k-1}<f(x)<c_{k+1}\right\}, \quad$ где $\quad k=\overline{1, n-1} \quad$ и $A_{n}=\left\{x \in X ; f(x)>c_{n-1}\right\}$. Заметим, что для любого $k=\overline{0, n}$ множества $A_{k}$ имеют тип $F_{\sigma}$. Тогда для пространства $X=\bigcup_{k=0}^{n} A_{k}$ по лемме 7 существуют множества $B_{0}, \ldots, B_{n}$ типа $F_{\sigma}$, такие, что $X=\coprod_{k=0}^{n} B_{k}, B_{k} \subset A_{k}$ для любого $k=\overline{0, n}$.

Введем функцию $f_{n}: X \rightarrow \mathbb{R}$, полагая $f_{n}(x)=c_{k}$ при $x \in B_{k}$ для любого $k=\overline{0, n}$. Согласно лемме $8, f_{n} \in B_{1}(X)$. Зафиксируем точку $x \in X$. Тогда для некоторого номера $k$ имеем, что $x \in B_{k} \subset A_{k}$. Значит, $f_{n}(x)=c_{k}$ и $c_{k-1}<f(x)<c_{k+1}$. Отсюда ясно, что $\left|f_{n}(x)-f(x)\right|<1 / n$.

Таким образом, при $n \rightarrow \infty$ функции $f_{n}$ равномерно сходятся к функции $f$ и, стало быть, функция $f(x) \in B_{1}(X)$.

Пусть теперь для любого открытого множества $U \subset \mathbb{R}$ его прообраз $f^{-1}(U)$ имеет тип $F_{\sigma}$ и функция $f$ неогораниченная. Рассмотрим функцию $g(x)=\operatorname{arctg} f(x)$. Очевидно, что для любого открытого множества $U \subset \mathbb{R}$ прообраз $g^{-1}(U)$ есть множество типа $F_{\sigma}$. Функция $g$ ограниченная и, следовательно, по предыдущему рассуждению является функцией первого класса Бэра. Тогда $f(x)=\operatorname{tg}(g(x))$ есть функция первого класса Бэра.

Теорема 10. Пусть пространство $X$ наследственно линделефово и $f: X \rightarrow \mathbb{R}$. Если на всяком непустом замкнутом множестве $F$ имеются точки непрерывности индуцированной функции $\left.f\right|_{F}$, то $f \in B_{1}(X)$.

Доказательство. В силу теоремы 9 достаточно показать, что прообраз открытого множества имеет тип $F_{\sigma}$.

Пусть $p, q \in \mathbb{R}, p<q$. Положим $P=\{x \in X ; f(x)>p\}, Q=\{x \in X ; f(x)<q\}$. Тогда $X=P \cup Q$. Используя метод трансфинитной индукции, определим $\omega_{1}$-последовательности замкнутых множеств $\left\{F_{\beta} \subset X ; \beta<\omega_{1}\right\}$ и открытых множеств $\left\{U_{\beta} ; \beta<\omega_{1}\right\}$, таких, что $F_{\alpha} \subset F_{\beta}$ при $\beta<\alpha$ и множество $f\left(U_{\beta} \cap F_{\beta}\right)$ принадлежит $P$ или $Q$ при любом $\beta<\omega_{1}$. Пусть $F_{0}=S_{A}$ и $U_{0}$ - окрестность некоторой точки непрерывности функции $f$, такая, что $f\left(U_{0}\right)$ принадлежит $P$ или $Q$. 
Предположим, что для всех $\beta<\alpha$ определены семейство открытых множеств $\left\{U_{\beta}\right\}_{\beta<\alpha}$ и семейство замкнутых множеств $\left\{F_{\beta}\right\}_{\beta<\alpha}$, таких, что $F_{\beta}=F_{\beta-1} \backslash U_{\beta-1}$, $U_{\beta-1}$ - окрестность некоторой точки непрерывности функции $\left.f\right|_{F_{\beta-1}}$, такая, что $f\left(U_{\beta-1}\right)$ принадлежит $P$ или $Q$, если $\beta$ - непредельный ординал и $F_{\beta}=\bigcap_{\gamma<\beta} F_{\gamma}$, $U_{\beta}$ - окрестность некоторой точки непрерывности функции $\left.f\right|_{F_{\beta}}$, такая, что $f\left(U_{\beta} \cap F_{\beta}\right)$ входит в $P$ или $Q$, если $\beta$ - предельный ординал. Если $\alpha$ такое, что существует $\alpha-1$, положим $F_{\alpha}=X \backslash\left(U_{0} \cup \ldots \cup U_{\alpha-1}\right)$. Заметим, что $F_{\alpha}=F_{\alpha-1} \backslash U_{\alpha-1}$ и $F_{\alpha} \subseteq F_{\alpha-1}$. Если $\alpha-$ предельный ординал, то $F_{\alpha}=\bigcap_{\beta<\alpha} F_{\beta}$. Пусть $U_{\alpha}$ - окрестность точки непрерывности функции $\left.f\right|_{F_{\alpha}}$, такая, что $f\left(U_{\alpha} \cap F_{\alpha}\right)$ входит в $P$ или $Q$. Нетрудно видеть, что семейства множеств $\left\{F_{\beta} \subset X ; \beta<\omega_{1}\right\}$ и $\left\{U_{\beta} ; \beta<\omega_{1}\right\}$ удовлетворяют требуемым условиям.

Пусть $G=X \backslash\left(\bigcap_{\alpha<\omega_{1}} F_{\alpha}\right)=\bigcup_{\alpha<\omega_{1}}\left(X \backslash F_{\alpha}\right)$. Так как пространство $X$ - наследственно линделефово, то существует ординал $\gamma<\omega_{1}$, такой, что $G=\bigcup_{\alpha<\gamma}\left(X \backslash F_{\alpha}\right)$ и $F_{\gamma}=F_{\gamma+1}=\ldots$. Если $F_{\gamma} \neq \varnothing$, то $U_{\gamma} \cap F_{\gamma} \neq \varnothing$, что невозможно, так как $F_{\gamma+1}=F_{\gamma} \backslash U_{\gamma}=F_{\gamma}$. Таким образом, существует ординал $\alpha<\omega_{1}$, такой, что $F_{\beta} \neq \varnothing$ при любом $\beta<\alpha \quad$ и $F_{\alpha}=\varnothing$. Тогда $X=\bigcup_{\beta<\alpha}\left(F_{\beta} \backslash F_{\beta+1}\right)$. Положим $T=\left\{\beta<\alpha ; F_{\beta} \backslash F_{\beta+1} \subset P\right\}, L=\left\{\beta<\alpha ; F_{\beta} \backslash F_{\beta+1} \subset Q\right\} \backslash T$. Ясно, что $L \cap T=\varnothing$. Тогда $X=A \cup B$, где $A=\bigcup_{\beta \in T}\left(F_{\beta} \backslash F_{\beta+1}\right), B=\bigcup_{\beta \in L}\left(F_{\beta} \backslash F_{\beta+1}\right)$. Поскольку для любого $\beta$ множества $F_{\beta} \backslash F_{\beta+1}$ имеют тип $F_{\sigma}$, то и множества $A$ и $B$ имеют тип $F_{\sigma}$. Заметим, что $A \subset P, B \subset Q$ и $A \cap B=\varnothing$.

Таким образом, для любых чисел $p, q \in \mathbb{R}$, где $p<q$, верно, что $X=A \cup B$, $A \subset P, B \subset Q$, множества $A, B$ имеют тип $F_{\sigma}$. Фиксируем число $p \in \mathbb{R}$ и последовательность $q_{1}>q_{2}>\ldots$, такую, что $p=\lim _{n \rightarrow \infty} q_{n}$. Для каждого $n \in \mathbb{N}$ $X=A_{n} \cup B_{n}$, где $A_{n} \cap B_{n}=\varnothing$, множества $A_{n}$ и $B_{n}$ имеют тип $F_{\sigma}, A_{n} \subset P$, $B_{n} \subset Q_{n}$, где $Q_{n}=\left\{x \in X ; f(x)<q_{n}\right\}$. Положим $R=\bigcup_{n=1}^{\infty} A_{n}$ и $W=\bigcap_{n=1}^{\infty} B_{n}$. Тогда $R \cap W=\varnothing, X=R \cup W$, множество $R$ имеет тип $F_{\sigma}$. Покажем, что $P=R$. Пусть $x \in P$. Тогда найдется число $n \in N$, такое, что $f(x)>q_{n}$ и $x \notin B_{n}$, Значит, $x \notin W$ Следовательно, $x \in R$. Таким образом, множество $P$ имеет тип $F_{\sigma}$. Аналогичный результат справедлив для множества $Q$. 
Следовательно, прообраз любого открытого множества имеет тип $F_{\sigma}$.

Таким образом, из следствия 5 и теоремы 10 получен следующий критерий для функций первого класса Бэра.

Теорема 11. Пусть пространство $X$ наследственно линделефово и наследственно бэровское. Функция $f: X \rightarrow \mathbb{R}$ принадлежит $B_{1}(X)$ тогда и только тогда, когда для любого непустого замкнутого подмножества $F \subset X$ функция $\left.f\right|_{F}$ имеет точку непрерывности.

В работе [9] для любого множества $A \subset \mathbb{R}$ определено пространство Хаттори $H(A)$. Это множество вещественных чисел $\mathbb{R}$, в котором база окрестностей точки $x$ определяется следующим образом:

$$
\begin{gathered}
\{(x-\varepsilon, x+\varepsilon), \forall \varepsilon>0\}, \text { если } x \in A \subset \mathbb{R} ; \\
\{[x, x+\varepsilon), \forall \varepsilon>0\}, \text { если } x \in \mathbb{R} \backslash A .
\end{gathered}
$$

Заметим, что пространства $S_{A}$ и пространства Хаттори $H(A)$ удовлетворяют условию теоремы 11 (см. [2]) и, значит, верно следующее

Следствие 12. Функция $f: E \rightarrow \mathbb{R}$, где $E=S_{A}$ или $E=H(A)$, принадлежит $B_{1}(E)$ тогда и только тогда, когда для любого непустого замкнутого подмножества $F \subset X$ функция $\left.f\right|_{F}$ имеет точку непрерывности.

Благодарность: Выражаю глубокую благодарность своему научному руководителю Хмылевой Татьяне Евгеньевне за ценные советы для выполнения данного исследования.

\section{ЛИТЕРАТУРА}

1. Александров П.С. Введение в теорию множеств и общую топологию. М.: Наука, 1977. $368 \mathrm{c}$.

2. Bouziad A., Sukhacheva E. On Hattori spaces // Commentationes Mathematicae Universitatis Carolinae. 2017. No. 2. P. 213-223. DOI 10.14712/1213-7243.2015.199.

3. van Douwen E.K. Closed copies of the rationals // Commentationes Mathematicae Universitatis Carolinae. 1987. V. 28. No. 1. P. 137-139.

4. Натансон И.П. Теория функций вещественной переменной. М.: Наука, 1974. 480 с.

5. Neubrunnová A. On quasicontinuous and cliquish functions // Časopis pro pěstování matematiky. 1974. V. 99. No. 2. P. 109-114.

6. Osipov A.V., Pytkeev E.G. On sequential separability of functional spaces // Topology and its Applications. 2017. V. 221. P. 270-274.

7. Tkachuk V.V. A Cp-Theory problems book. Topological and functions space. New York: Springer, 2011.

8. Энгелькинг Р. Общая топология. М.: Мир, 1986. 752 с.

9. Hattori Y. Order and topological structures of posets of the formal balls on metric spaces // Mem. Fac. Sci. Eng. Shimane Univ. Ser. B Math. Sci. 2010. V. 43. P. 13-26.

Статья поступила 13.04.2018 г.

Sukhacheva E.S. (2018) ON FIRST BAIRE CLASS FUNCTIONS DEFINED ON SOME CLASSES OF NONMETRIZABLE SPACES. Vestnik Tomskogo gosudarstvennogo universiteta. Matematika i mekhanika [Tomsk State University Journal of Mathematics and Mechanics]. 53. pp. $39-46$

DOI $10.17223 / 19988621 / 53 / 4$ 
For first Baire class functions given on Polish spaces, Baire's and Lebesgue's criteria are known. We prove analogs of these theorems for topological spaces that are both hereditarily Lindelöf and hereditarily Baire spaces.

An analogue of Lebesgue's theorem is as follows: let a space $X$ be a hereditarily Lindelöf space and a function $f: X \rightarrow \mathbb{R}$. A function $f$ is a first Baire class function if and only if the inverse image of an open set in $\mathbb{R}$ has type $F_{\sigma}$.

The necessity of the following theorem is true for hereditarily Baire spaces and the proof uses the concept of cliquish functions. We affirm that sufficiency is true for hereditarily Lindelöf spaces.

An analogue of Baire's theorem is as follows: let $X$ be a hereditarily Lindelöf and hereditarily Baire space. A function $f: X \rightarrow \mathbb{R}$ belongs to the set of first Baire class functions if and only if for any non-empty closed subset $F$ the function $\left.f\right|_{F}$ has a point of continuity.

For a subset $A$ of the real line $\mathbb{R}$, a modification of the Sorgenfrey line $S$ denoted as $S_{A}$ is defined as follows: neighborhoods of points from $A$ are given by neighborhoods of the right halfopen topology, and those in the complement of $A$ are given by neighborhoods of the left halfopen topology. For a subset $A$ of the real line $\mathbb{R}$, a Hattori space denoted as $H(A)$ is defined as follows: neighborhoods of points from $A$ are given by usual Euclidean neighborhoods and those in the complement of $A$ are given by neighborhoods of the right half-open topology. In particular, spaces $S=S_{\varnothing}, S_{A}$, and $H(A)$ satisfy the conditions of the previous two theorems.

Keywords: Sorgenfrey line, function of the first Baire class, hereditarily Baire space, hereditarily Lindelöf space, cliquish function, $F_{\sigma}$ and $G_{\delta}$ sets.

AMS Mathematical Subject Classification: 54E52, 26A21

SUKHACHEVA Elena Sergeevna (Postgraduate student faculty of mechanics and mathematics, Tomsk State University, Tomsk, Russian Federation; Département de Mathématiques, Université de Rouen, UMR CNRS 6085, Avenue de l'Universite, BP.12, F76801 Saint-Etienne-du-Rouvray, France). E-mail: sirius9113@mail.ru

\section{REFERENCES}

1. Aleksandrov P.S. (1977) Vvedenie v teoriyu mnozhestv i obshchuyu topologiyu [Introduction to the set thory and general topology]. Moscow: Nauka. $368 \mathrm{p}$.

2. Bouziad A., Sukhacheva E. (2017) On Hattori spaces. Commentationes Mathematicae Universitatis Carolinae. 2. pp. 213-223. DOI 10.14712/1213-7243.2015.199.

3. van Douwen E.K. (1987) Closed copies of the rationals. Commentationes Mathematicae Universitatis Carolinae. 28(1). pp. 137-139.

4. Natanson I.P. (1974) Teoriya funktsiy veshchestvennoy peremennoy [Theory of functions of a real variable]. Moscow: Nauka. $480 \mathrm{p}$.

5. Neubrunnová A. (1974) On quasicontinuous and cliquish functions. Časopis pro pěstování matematiky. 99(2). pp. 109-114.

6. Osipov A.V., Pytkeev E.G. (2017) On sequential separability of functional spaces. Topology and its Applications. 221. pp. 270-274. https://doi.org/10.1016/j.topol.2017.02.059.

7. Tkachuk V.V. (2011) A $C_{p}$-Theory problems book. Topological and functions space. New York: Springer.

8. Engelking R. (1977) General Topology. Warszawa: PWN Publ.

9. Hattori Y. (2010) Order and topological structures of posets of the formal balls on metric spaces. Mem. Fac. Sci. Eng. Shimane Univ. Ser. B Math. Sci. 43. pp. 13-26. 